\title{
ASYMMETRY IN REGARD TO THE EFFECTS OF MAGNOCELLULAR AND PARVOCELLULAR LESIONS
}

\author{
Bernt Christian Skottun \\ Ullevaalsalleen $4 \mathrm{C}$ \\ 0852 Oslo \\ Norway \\ e-mail: berntchrskottun@gmail,com
}

Short title: Magno/parvo asymmetry

\begin{abstract}
The placing of lesions in the magno- and parvocellular layers of the Lateral Geniculate Nucleus (LGN) of the visual stream has been used in attempts to assess the contributions of the two systems to various visual tasks. However, because there are about ten times as many parvocellular cells as magnocellular cells a lesion blocking the parvocellular input would be expected to have a larger deleterious impact than one blocking the magnocellular input. Thus, a visual task that depends upon all inputs, i.e. which is not linked specifically to either the magno- or parvocellular systems, would be expected to be more severely affected by a lesion in the parvocellular system than by one in the magnocellular system simply on the basis of the number of cells involved. A larger impact of a parvocellular lesion can, therefore, not be taken to mean that the task in question is specifically, or predominantly, linked to this system. Effects following magnocellular lesions (and not observed following parvocellular lesions), on the other hand, cannot be accounted for on the basis of cell number. There is, therefore, an asymmetry, in regard to the significance of the effects of lesions placed in the magno- and parvocellular layers of the LGN.
\end{abstract}

Key words: Vision; lesions; magnocellular; parvocellular; number of cells; texture; orientation. 
The visual pathways stretching from the eyes to the primary visual cortex can be differentiated into (at least) two parallel streams: The magno- and the parvocellular systems (Merigan \& Maunsell, 1994; Schiller \& Logothetis, 1990; Shapley \& Perry, 1986). A number of attempts have been made at identifying psychophysical tasks that make it possible to assess the integrity of these systems. Most of these attempts have focused on the magnocellular system [FOOTNOTE 1]. Attempts at finding tasks to assess parvocellular integrity are by comparison relatively few. However, recently Taylor-Cooke et al. (2021) sought to use "texture detection" to assess parvocellular integrity. This attempt was based on the observations of Schiller et al. (1990) who found that lesions placed in the parvocellular layers of the Lateral Geniculate Nucleus (LGN) in monkeys had a profound deleterious effect upon texture discrimination whereas lesions placed in the magnocellular layers had no effect upon performance on this task (see Fig. 21 of Schiller et al., 1990). The interpretation of these observations by Taylor-Cooke et al. (2021), it would seem, stands in need some comments.

Orientation selectivity. It appears Taylor-Cooke et al. (2021) modeled their stimulus after the one used by Schiller et al. (1990). Taylor-Cooke et al. (2021; p. 3) wrote "Tasks used in this study were developed on the basis of Schiller and colleagues [referring here to Schiller et al., 1990]" which, according to Taylor-Cooke et al. (2021, p. 3) "showed that TD [Texture Detection] is mediated exclusively within the PC [Parvocellular] system". The stimulus used by Schiller et al. (1990)(which is depicted in Fig. 1 of their paper) consisted of a field of small tilted line segments. The task was to detect an area in this field in which the line segments had the orientation orthogonal to those in the rest of the field. In regard to their stimuli Taylor-Cooke et al. (2021, p. 3) wrote: "The visual stimulus was a field of dark gray, diagonal dashed lines ..." in which "[o]ne of the eight locations will have dashed lines going in the opposite direction". It seems that by "opposite direction" they meant "opposite orientation" or "orthogonal orientation". It appears reasonable to assume that their stimulus was very similar to the one used by Schiller et al. (1990).

It would therefore seem that the task given to subjects by Taylor-Cooke et al. (2021) was one of discriminating between orientations, i.e. discriminating an area of line segments oriented in one direction from an area in which the line segments were oriented in a different orientation. The problem in this connection is that parvocellular cells (as well as magnocellular cells) are essentially insensitive to orientation. It would seem problematic to use a task based on orientation to test the integrity of a part of the visual system that is not sensitive to orientation. 
In the case of the results of Schiller et al. (1990) it may be that lesions in the parvocellular layers abolished or reduced the input to orientation selective cells (such as those in the primary visual cortex). In which case, in testing "Texture Detection" in the way carried out by Taylor-Cooke et al. (2021) one would not be able tell if one were testing the state of the parvocellular input or the state of the orientation selective units to which the parvocellular system is providing the input. The lesson to be taken from this is that when destruction of some neuronal entity causes a reduction in performance on some particular task that does not mean that the destructed structure is the one that carries out the task as it is possible that it only provides the input to the critical structure. This brings us to the next and the more important point.

Number of cells. As was just pointed out, it may be that lesions of the parvocellular layers simply abolished the input to orientation selective cells in the cortex. In this connection it should be noted that there are approximately ten times as many parvocellular cells as there are magnocellular cells (Ahmad and Spear, 1993; Peters et al., 1994). If the lesions made by Schiller at al. (1990) abolished all input from a given subsystem for a particular area of visual space we would have to expect that parvocellular lesions reduced input to the visual cortex by an amount ten times larger than did corresponding lesions in the magnocellular layers. Consequently, in the case of a given visual task which relies upon all incoming inputs (e.g., which simply summed all inputs) and which is not linked specifically to either the parvo- or magnocellular systems one would expect lesions in the parvocellular system to have a much larger effect than lesions in the magnocellular system. Also, because parvocellular cells provide the bulk of the input to the visual cortex one would expect parvocellular lesions to cause reduced performance on a large variety of visual tasks. That is exactly what Schiller et al. (1990) found: Pattern discrimination, brightness discrimination, shape discrimination, color discrimination, stereo detection, luminance contrast detection, and texture discrimination were all substantially reduced following the placing of parvocellular lesions. In the case of magnocellular lesions owing to the smaller size of the magnocellular input one would expect the effect of lesions to this system to be relatively minor. Consistent with this Schiller et al. (1990) found the effects of magnocellular lesions on the mentioned tasks to be generally modest.

The issue of number of cells may take on more significance if there were a sigmoidal relationship between the number of input cells and performance. Such a saturating relationship could occur if some factor other than the number of cells were setting the upper limit to performance. In such a case blocking of all magnocellular input would lead to an approximately $10 \%$ reduction in input from $100 \%$ to $90 \%$. This 
would lead to little or no reduction in performance since a sigmoidal curve is relatively flat at the upper end. On the other hand, the blocking of all parvocellular input would give a reduction from $100 \%$ to about 10 $\%$ of the input which, we would have to expect, would result in a pronounced performance reduction. This scenario is illustrated in Fig. 1.

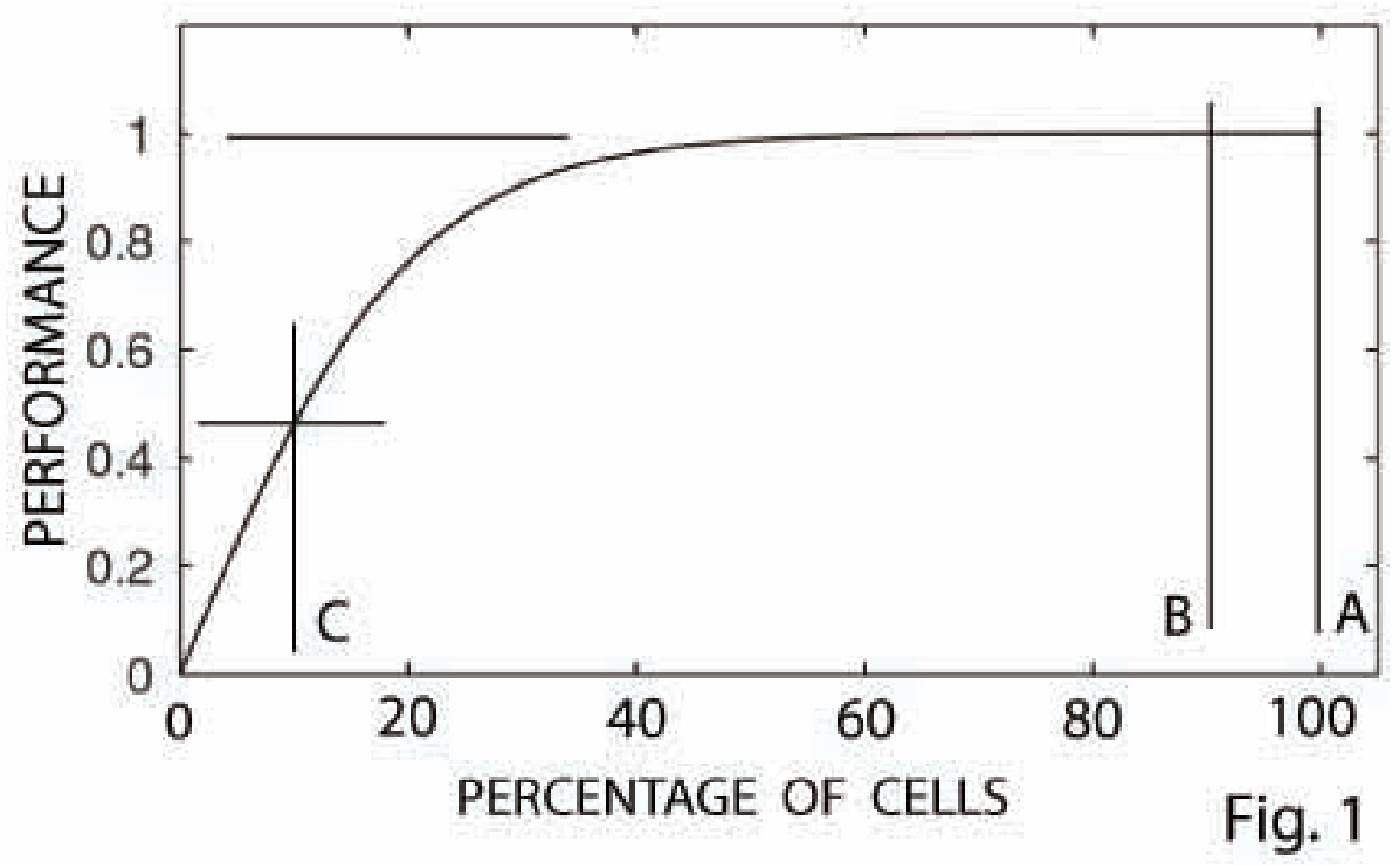

Fig. 1. The theoretical effect of reduced cell number in the case of a sigmoidal relationship between the number of input cells and visual performance. In the case of a magnocellular lesion, the blocking of all magnocellular input would result in a reduction of about $10 \%$ in overall input from $100 \%$ to $90 \%$ (going from the vertical line marked "A" to the one marked "B") which would cause practically no reduction in performance. However, the blocking of all parvocellular inputs would cause a $90 \%$ reduction in overall input from $100 \%$ to only $10 \%$. (i.e., going from the vertical line marked "A" to the one labeled "C"). This would result in a pronounced reduction in performance. Thus, it would be possible for parvocellular lesions to have large impact on some given visual task and for magnocellular lesions to have no impact on the task without the task in question being specifically linked to the parvocellular system.

Conclusions. It would seem that the kind of test carried out by Taylor-Cooke et al. (2021) would/could reflect activity in orientation selective cells in the cortex and that these cells need not be linked specifically to the parvocellular system. However, the wider and more important consequence of the present observations is that 
there is an asymmetry in regard to the results obtained with lesions placed in the parvo- and magnocellular layers of the LGN. In the case of parvocellular lesions any reduction in performance on a given task (e.g., accompanied by only a small or no performance reduction following magnocellular lesioning) could simply be the result of an unspecific reduction in the amount of input. That is, it need not mean that the task is specifically linked to the parvocellular system as it could simply reflect a larger impact of a parvocellular lesion reflecting the larger number of cells in this system. On the other hand, were one to find a reduction in performance on some task following magnocellular lesions and that a lesion in the parvocellular system had no or little impact on performance one may rule out that the effect is the result of a reduction in overall input. There exists, therefore, an asymmetry between the significance of the effects of magno- and parvocellular lesions. In summary: The effects of lesions in the magno- and parvocellular systems are not equivalent.

\section{FOOTNOTE}

1. This is a large topic that cannot be addressed in a brief note such as the present one. However, one issue ought to be mentioned as it has been the source of some confusion. A number of authors have proposed that using stimuli of low spatial frequency may preferentially activate the magnocellular system. A review of this issue has revealed that the magno- and parvocellular systems are quite similar in regard to how their responses depend on stimulus spatial frequency (Skottun, 2015). Thus, it seems problematic to use spatial frequency in stimuli at supra threshold contrasts to separate contributions from the two systems. However, there is ample evidence that contrast detection (i.e., contrast threshold) of low spatial frequency stimuli is mediated by the magnocellular system. (For references see Skottun, 2000.) Thus, it may be possible to use spatial frequency to isolate the contribution from the magnocellular system at contrast threshold but not at suprathreshold contrasts (Skottun, 2015). 


\section{REFERENCES}

Ahmad, A., \& Spear, P.D. (1993) Effects of aging on the size, density, and number of Rhesus monkey lateral geniculate neurons. Journal of Comparative Neurology, 334, 631-643.

Merigan, W.H., \& Maunsell, J.H. (1994) How parallel are the primate visual pathways? Annual Review of Neuroscience, 16, 369-402.

Peters, A., Payne, B.R., \& Budd, J. (1994) A numerical analysis of the geniculocortical input to striate cortex in the monkey. Cerebral Cortex 4, 215-229.

Schiller, P.H., \& Logothetis, N.K. (1990) The color-opponent and broad-band channels of the primate visual system. Trends in Neurosciences, 13, 392-398.

Schiller, P.H., Logothetis, N.K., \& Charles, E.R. (1990) Role of the color-opponent and broad-band channels in vision. Visual Neuroscience, 7, 3416-3468.

Shapley, R., \& Perry, V.H. (1986) Cat and monkey retinal ganglion cells and their visual functional roles. Trends in Neurosciences, 9, 229-235.

Skottun, B.C. (2000) The magnocellular deficit theory of dyslexia: the evidence from contrast sensitivity. Vision Research, 40, 111-127.

Skottun, B.C. (2015). On the use of spatial frequency to isolate contributions from the magnocellular and parvocellular systems and the dorsal and ventral cortical streams. Neuroscience and Biobehavioral Reviews, $56,266-275$.

Taylor-Cooke, P.A., Chacko, J.G., Chelette, K., \& Mennemeier, M.S. (2021) Lateralized Effects in Troxler Fading and Parvo and Magnocellular Tasks after Localized 1Hz rTMSs. Frontiers in Neurology and Neuroscience Research, 2, 100013. 